\title{
Biased Beliefs about Immigration, Economic Concerns, and Information Provision: The Role of Cultural Distance* \\ Pre-Analysis Plan
}

\author{
Patrick Bareinz ${ }^{\dagger}$ \\ Silke Uebelmesser $\ddagger$ \\ University of Jena \\ University of Jena and CESifo
}

September 22, 2021

\begin{abstract}
We investigate whether individual perceptions of the origin of immigrants are linked to economic concerns about immigration in the host society. In our experiment, participants are randomly assigned to one of three experimental groups: two treatment arms and a passive control group. While respondents in the first treatment arm are only subject to belief elicitation employed as a priming device, respondents in the second treatment arm are exposed to factual information about the overall share of immigrants in their society as well as the share of immigrants stemming from European countries. Conversely, the passive control group is neither exposed to factual information nor the priming device. We aim to analyze whether information provision and/or the priming treatment translate into economic concerns about immigration, immigration policy preferences and preferences for redistribution.
\end{abstract}

JEL classification: C90, D83, F22, H20, J15.

Keywords: immigration attitudes, survey experiment, information provision, belief updating, welfare state, labor market, preferences for redistribution.

*This pre-analysis plan describes an experiment which builds on the RCT registered under ID: AEARCTR0006819. For the initial RCT and pre-analysis plans, please refer to www.socialscienceregistry.org/trials/6819. This work is supported by the Free State of Thuringia and the European Social Fund. IRB approval was obtained at FSU Jena.

'University of Jena, Carl-Zeiss-Str. 3, 07743 Jena, Germany, email: patrick.bareinz@uni-jena.de.

‡University of Jena, Carl-Zeiss-Str. 3, 07743 Jena, Germany, email: silke.uebelmesser@uni-jena.de. 


\section{Motivation}

The experiment described in this pre-analysis plan is complementary to the RCT registered under ID: AEARCTR-0006819 (Bareinz and Uebelmesser 2020). ${ }^{1}$ The experimental design described in the following extends the RCT in Bareinz and Uebelmesser (2020) by directly adressing individual beliefs about cultural distance between the immigrant population and the host society.

\section{Experimental Design}

Our experimental design consists of four stages and three experimental groups.

\subsection{Elicitation of prior beliefs}

First stage (for respondents allocated to either treatment arm I or II):

- Elicitation of respondents' prior beliefs about the share of immigrants in Germany

- Elicitation of respondents' prior beliefs about the share among yearly immigrants stemming from European countries in Germany ${ }^{2}$

\subsection{Treatment arms}

Second stage:

- Treatment arm I: respondents are not provided with factual information about immigration statistics. For this group, the treatment consists of belief elicitation in stage 1 employed as a priming device.

- Treatment arm II: Respondents are provided with factual information about the overall share of immigrants as well as the share of immigrants stemming from European countries, after having stated their beliefs in stage 1 .

\footnotetext{
${ }^{1}$ For the corresponding pre-analysis plans, please refer to: www.socialscienceregistry.org/trials/6819

${ }^{2}$ The definition of European countries follows the German Federal Office for Migration and Refugees in that it includes immigrants from Turkey and Russia. Respondents are provided with this definition when stating their beliefs.
} 
- Control group: respondents are neither exposed to factual information nor to belief eliciation prior to the measurement of outcomes.

The information treatment in treatment arm II further contains conditional feedback on respondents' prior beliefs, stating whether a respondent has underestimated, correctly estimated $^{3}$, or overestimated the respective statistic. The wording of our information treatment for treatment arm II is presented in the following: ${ }^{4}$

\section{- Treatment arm II:}

"We will take a brief look at your two estimates:

The share of immigrants in Germany is around 13 percent. Your estimate of [show estimate] was therefore [too low / quite accurate / too high].

The share of European immigrants in Germany is around 66 percent. Your estimate of [show estimate] was therefore [too low / quite accurate / too high]".

Concerning the two pieces of information contained in treatment $I I$, the overall share of immigrants represents the size of the immigrant population in the host society. In addition, we assume that the share of immigrants from European countries is perceived as a signal for aggregate cultural distance to the immigrant population.

\subsection{Outcome variables}

Third stage:

Respondents are asked literature-based survey measures of attitudes towards immigration and preferences for redistribution:

- Welfare state and labor market concerns about immigration (economic channels)

- Immigration policy preferences

- Preferences for redistribution

\footnotetext{
${ }^{3}$ We allow for a margin of error of \pm 1 percentage points for respondents to receive the feedback of correct estimation.

${ }^{4}$ In addition to the information treatment itself, we disclose the sources of the information provided to signal its credibility: while the information on the share of immigrants stems from the German Federal Statistical Office, the information on the share of immigrants stemming from European countries stems from the German Federal Office for Migration and Refugees.
} 
For our outcome variables, we employ the measures used in Bareinz and Uebelmesser (2020). Their wording is presented in the following:

- Welfare state concerns: "Immigrants pay taxes and receive social benefits from the health care and social insurance systems. On balance, do you think that immigrants in Germany receive more social benefits than they pay taxes, or that they pay more taxes than they receive social benefits?". Answers range from 0 for "Receive more social benefits" to 10 for "Pay more taxes".

- Labor market concerns: "Do you think that immigrants rather take away jobs from workers in Germany, or that they rather help to create new jobs?". Answers range from 0 for "Take jobs away" to 10 for "Create new jobs".

- Immigration policy preferences: “Do you think that the number of immigrants coming to Germany each year should be: decreased a lot / decreased slightly / stay the same / increased slightly / increased a lot?".

- Preferences for redistribution: "Some people think that the government should not care about income differences between rich and poor people. Others think that the government should do everything in its power to reduce income inequality. What do you think?". Answers range from 0 for "Government should not care about income inequality" to 10 for "Government should do everything against income inequality".

- Preferences for income support programs: "Would you say that you favor or oppose an increase in income support programs for poor people? Keep in mind that, in order to finance such an increase other types of governmental spending (like spending on infrastructure and defense, for example) would have to be scaled down, government debt would have to increase or taxes would have to be raised." Answers range from 0 for "Strongly oppose" to 10 for "Strongly favor".

- Preferences for governmental support programs conditional on nationality: "Some people think that the government should only support people who have German citizenship. Others think that the government should care equally about all people in Germany, regardless of their citizenship. What do you think?". Answers range from 0 for "Govern- 
ment should focus on people with German citizenship" to 10 for "Government should care equally about all people".

In addition, we employ a secondary outcome measure: an ex post assessement of advantages and disadvantages of immigration for the host society within the last 10 years. This outcome variable is worded as follows:

- Ex post assessment of immigration: "What would you say: Do you think immigrants have created more disadvantages or more advantages for Germany in the last 10 years?". Answers range from 0 for "Have created more disadvantages" to 10 for "Have created more advantages".

All of our outcome variables are coded such that a higher value indicates a more positive attitude towards immigration or a more supportive attitude towards redistribution, respectively. Labor market concerns, welfare state concerns, and preferences for redistribution are measured on an 11-point scale, while immigration policy preferences are measured on a 5-point scale.

\subsection{Elicitation of posterior beliefs}

Fourth stage (for respondents allocated to either treatment arm I or II):

- Elicitation of respondents' posterior beliefs about the share of immigrants in Germany

- Elicitation of respondents' posterior beliefs about the share of immigrants stemming from European countries in Germany

Fourth stage (for respondents allocated to the passive control group:

- Elicitation of respondents' beliefs about the share of immigrants in Germany

- Elicitation of respondents' beliefs about the unemployment rate of immigrants in Germany

- Elicitation of respondents' beliefs about the share of immigrants stemming from European countries in Germany

The elicitation of (posterior) beliefs for all respondents allows us to (i) investigate whether respondents in treatment arm II engage in belief updating after the receipt of facts about 
immigration, and to (ii) evaluate the degree of belief updating between-subject, and relative to the beliefs in the passive control group.

\section{Main Hypotheses}

To derive our main hypotheses, we focus on the case in which respondents (i) overestimate the overall share of immigrants, but (ii) underestimate the share of immigrants stemming from European countries. In this setting, respondents' beliefs about the share of immigrants are hence positvely biased, while respondents' beliefs about the share of immigrants stemming from European countries are negatively biased, on average:

Hypothesis I - Welfare state channel: Information provision translates into a more positive assessment of immigrants' welfare state contribution and hence lower welfare state concerns when respondents learn about a smaller size of the immigrant population and a larger proportion of European immigrants than believed ex ante on average.

Hypothesis IIa - Labor market channel: scenario a.: Information provision translates into lower concerns of respondents about labor market competition when they learn about a smaller size of the immigrant population and a larger proportion of European immigrants than believed ex ante on average. In this scenario, the smaller size of the immigrant population is perceived as less current competition on the job market, while the larger proportion of European immigrants is not (or only to smaller extent) moderating this effect.

Hypothesis IIb - Labor market channel: scenario b.: Information provision translates into the same or larger concerns of respondents about labor market competition when they learn about a smaller size of the immigrant population and a larger proportion of European immigrants than believed ex ante on average. In this scenario, the larger proportion of European immigrants is perceived as a signal for larger potential competition on the job market, while the lower size of the immigrant population is, again, perceived as less current competition on the job market, potentially offsetting each other. 
Hypothesis III - Immigration policy preferences: Information provision translates into more positive immigration policy preferences of respondents when they learn about a smaller size of the immigrant population and a larger proportion of European immigrants than believed ex ante on average.

Hypothesis IV - Preferences for redistribution: Information provision translates into more supportive preferences for redistribution of respondents when they learn about a smaller size of the immigrant population and a larger proportion of European immigrants than believed ex ante on average.

\section{Data}

\subsection{Main survey}

Our experiment is embedded into a large-scale representative online survey of 7000 invidividuals in Germany. The survey is quota-representative with respect to age, gender, educational background, and residence in Eastern/Western Germany. The survey field phase starts in mid of September 2021 and is distributed to respondents by a professional survey company via an online panel.

With respect to the experiment described in this pre-analysis plan, a random subset of in expectation about 3000 individuals will be investigated.

\subsection{Follow-up survey}

In addition to our main survey, we will conduct a follow-up survey of 50 percent of the total sample size to examine beliefs over time. Concerning the experiment described in this preanalysis plan, we thus expect a follow-up sample size of about 1500 individuals. The analysis for the follow-up survey will be based on the analysis for the main survey data.

\section{Analysis}

In the following, we outline the different steps we aim to conduct in our empirical analysis. 


\subsection{Experimental balance}

We first assess experimental balance by conducting between-subject t-tests. ${ }^{5}$ Specifically, we aim to conduct balance tests on the following covariates:

- prior beliefs: share of immigrants / share of European immigrants

- concerns about immigration

- attitudes towards cultural diversity

- concerns about economic situation

- concerns about COVID-19 crisis

- news consumption

- risk and trust attitudes

- political attitude

- age group

- gender

- residence / Eastern and Western Germany

- education

- employment status

- household size and net income

- relationship status

- migration background and contact with foreigners

- population size of area of residence

${ }^{5}$ An alternative approach considered here is the assessment of experimental balance as normalized differences between groups, as introduced by Imbens (2015). This is of particular relevance for large sample sizes. 


\subsection{Determinants of prior beliefs}

We explore which determinants of respondents' characteristics are associated with biased beliefs about immigration facts. Specifically, we estimate the following equation:

$$
b_{i}=\delta_{0}+\delta^{T} X_{i}+\varepsilon_{i}
$$

where $b_{i}$ represents biases in beliefs about the share of immigrants and the share of European immigrants, respectively, $X_{i}$ contains the control variables used in the balance tests, and $\varepsilon_{i}$ is the error term.

\subsection{Belief updating and differences in learning}

To investigate whether individuals in treatment arm II update their beliefs after the receipt of information, we compare their prior and posterior beliefs by means of within-subject t-tests.

In addition, we examine the degree of belief updating between-subject by estimating the following equation, in which treatment arm I serves as the base group:

$$
p_{i}=\rho_{0}+\rho_{1} I I_{i}+\varepsilon_{i}
$$

where $p_{i}$ represents posterior beliefs about immigration statistics, $I I_{i}$ is the treatment indicator for treatment arm $I I$, and $\varepsilon_{i}$ is the error term.

We further examine whether there exist differences in stated (prior) beliefs between the passive control group and treatment arm $I$, which differ in terms of the timing of belief elicitation within the survey. We hence estimate the following equation, employing the passive control group as the base group:

$$
s_{i}=\rho_{0}+\rho_{1} I_{i}+\varepsilon_{i}
$$

where $s_{i}$ represents stated (prior) beliefs about immigration statistics, $I_{i}$ is the treatment indicator for treatment arm $I$, and $\varepsilon_{i}$ is the error term. 


\subsection{Global effects of information provision and priming}

To investigate the global, i.e. full-sample effects of our information and priming treatments, we estimate the following equation which compares our outcome variables across treatment arms given exogeneity of the treatments:

$$
y_{i}=\gamma_{0}+\gamma_{1} I_{i}+\gamma_{2} I I_{i}+\varepsilon_{i}
$$

where $y_{i}$ represents the outcome variable, $I_{i}, I I_{i}$ are treatment indicators for the two treatment arms, and $\varepsilon_{i}$ is the error term. This specification allows to examine effects of priming and information relative to the passive control group.

To further investigate effects of information relative to the priming treatment $\mathrm{arm}^{6}$, we estimate the following equation, employing treatment arm $I$ as the base group:

$$
y_{i}=\gamma_{0}+\gamma_{1} I I_{i}+\varepsilon_{i}
$$

where $y_{i}$ represents the outcome variable, $I I_{i}$ is the treatment indicator for treatment arm $I I$, and $\varepsilon_{i}$ is the error term. This specification allows to examine effects of information only, abstracting from priming effects to due belief elicitation.

\subsection{Treatment effect heterogeneity}

We expect treatment effects to be heterogeneous across different subgroups of the population of interest. To analyze this potential treatment effect heterogeneity, we follow a systematic approach based on a machine learning algorithm put forward by Athey and Imbens (2016, 2019) called causal tree analysis. To evaluate conditional average treatment effects (CATE) by means of this algorithm, we supply the covariates used in the balances tests as potential sources of heterogeneity.

Based on the resulting causal tree, we will then reestimate our main specfications in equations (4) and (5) for the most relevant subgroups in terms of CATE. In addition to the evaluation based on the causal tree, we specifically consider the analysis of potential differences for sub-

\footnotetext{
${ }^{6}$ Note that the priming treatment arm in our setting is identical to a traditional control group in many information provision experiments, as it does not receive any information but is subject to belief elicitation.
} 
groups based on residence in Eastern and Western Germany and based on the distribution of prior beliefs.

\subsection{Extensions to the priming analysis}

Drawing from the data collected in Bareinz and Uebelmesser (2020), we further aim to examine priming effects related to belief elicitation of the share and the unemployment rate of immigrants, following a similar strategy as described in section 5.4. For that purpose, the passive control group contained in the experiment described in this pre-analysis plan can serve as the base group to investigate whether belief elicitation in Bareinz and Uebelmesser (2020) influences respondents' attitudes towards immigration.

\subsection{Further strategies for analysis}

Since our experimental design involves multiple treatment arms and outcome variables, we discuss further related strategies for our empirical analysis in the following.

\subsubsection{Adressing potential imbalances}

In case the realized experimental groups exert imbalances despite randomization for some covariates, we will control for these imbalances in terms of observables in our estimation specifications.

\subsubsection{Pooling of data treatment arms}

Based on the related designs of the experiment described in this pre-analysis plan and Bareinz and Uebelmesser (2020), we consider pooling data and experimental groups from these two experiments, especially in the context of the evaluation of treatment effect heterogeneity.

\subsubsection{Indices}

The related nature of our outcome variables further allows for the construction of indice measures, e.g. based on the outcome variables related to immigration and redistribution, respec- 
tively. This strategy may again be well-suited to be applied in the context of the evaluation of heterogeneity in treatment effects across subgroups of the population. 


\section{References}

Athey, Susan and Guido W. Imbens (2016). “Recursive Partitioning for Heterogeneous Causal Effects". Proceedings of the National Academy of Sciences 113 (27), 7353-7360.

- (2019). "Machine Learning Methods That Economists Should Know About". Annual Review of Economics $11(1), 685-725$.

Bareinz, Patrick and Silke Uebelmesser (2020). Biased Beliefs about Immigration, Economic Concerns, and Information Provision. AEA RCT Registry: ID AEARCTR-0006819, available online: https://doi.org/10.1257/rct.6819-3.0.

Imbens, Guido W. (2015). "Matching Methods in Practice: Three Examples". Journal of Human Resources 50 (2), 373-419. 